ARTICLE

https://doi.org/10.1038/s41467-019-13066-9

\title{
Infrared nano-spectroscopy of ferroelastic domain walls in hybrid improper ferroelectric $\mathrm{Ca}_{3} \mathrm{Ti}_{2} \mathrm{O}_{7}$
}

\author{
K.A. Smith (1) ${ }^{1}$, E.A. Nowadnick ${ }^{2,3,4 \star}$, S. Fan ${ }^{5}$, O. Khatib (1) 6,7, S.J. Lim ${ }^{8,9}$, B. Gao (1) ${ }^{9}$, N.C. Harms (1) ${ }^{1}$, S.N. Neal', \\ J.K. Kirkland ${ }^{1}$, M.C. Martin (1) ${ }^{7}$, C.J. Won (10 ${ }^{10}$, M.B. Raschke ${ }^{6}$, S.-W. Cheong $8,9,10$, C.J. Fennie (1) ${ }^{3}$, G.L. Carr ${ }^{11}$, \\ H.A. Bechtel ${ }^{7} \&$ J.L. Musfeldt (iD) ${ }^{1,5 *}$
}

Ferroic materials are well known to exhibit heterogeneity in the form of domain walls. Understanding the properties of these boundaries is crucial for controlling functionality with external stimuli and for realizing their potential for ultra-low power memory and logic devices as well as novel computing architectures. In this work, we employ synchrotron-based nearfield infrared nano-spectroscopy to reveal the vibrational properties of ferroelastic ( $90^{\circ}$ ferroelectric) domain walls in the hybrid improper ferroelectric $\mathrm{Ca}_{3} \mathrm{Ti}_{2} \mathrm{O}_{7}$. By locally mapping the $\mathrm{Ti}-\mathrm{O}$ stretching and $\mathrm{Ti}-\mathrm{O}-\mathrm{Ti}$ bending modes, we reveal how structural order parameters rotate across a wall. Thus, we link observed near-field amplitude changes to underlying structural modulations and test ferroelectric switching models against real space measurements of local structure. This initiative opens the door to broadband infrared nanoimaging of heterogeneity in ferroics.

\footnotetext{
${ }^{1}$ Department of Chemistry, University of Tennessee, Knoxville, TN 37996, USA. ${ }^{2}$ Department of Physics, New Jersey Institute of Technology, Newark, NJ 07102, USA. ${ }^{3}$ School of Applied and Engineering Physics, Cornell University, Ithaca, NY 14850, USA. ${ }^{4}$ Department of Materials Science and Engineering, University of California, Merced, Merced, CA 95343, USA. ${ }^{5}$ Department of Physics, University of Tennessee, Knoxville, TN 37996, USA. ${ }^{6}$ Department of Physics, Department of Chemistry, and JILA, University of Colorado, Boulder, CO 80309, USA. ${ }^{7}$ Advanced Light Source Division, Lawrence Berkeley National Laboratory, Berkeley, CA 94720, USA. ${ }^{8}$ Rutgers Center for Emergent Materials, Rutgers University, Piscataway, NJ 08854, USA. ${ }^{9}$ Department of Physics and Astronomy, Rutgers University, Piscataway, NJ 08854, USA. ${ }^{10}$ Laboratory for Pohang Emergent Materials, Pohang Accelerator Laboratory and Max Planck POSTECH Center for Complex Phase Materials, Pohang University of Science and Technology, Pohang 790-784, Korea. ${ }^{11}$ National Synchrotron Light Source II, Brookhaven National Laboratory, Upton, NY 11973, USA. *email: enowadnick@ucmerced.edu; musfeldt@utk.edu
} 
$\mathrm{M}$ any unique properties in functional materials arise from spatially heterogeneous electronic and magnetic states. Examples include the formation of charge and spin stripes in some cuprates and nickelates and phase separation across the Mott transition of $\mathrm{VO}_{2}{ }^{1-7}$. Ferroics also have naturally occurring heterogeneities in the form of domains and domain walls ${ }^{8-18}$. This is especially true for $\mathrm{Ca}_{3} \mathrm{Ti}_{2} \mathrm{O}_{7}$, a hybrid improper ferroelectric ${ }^{19,20}$ where the polarization arises from a trilinear coupling mechanism ${ }^{21-23}$ and abundant charged domain walls have been observed ${ }^{19,20,24}$. Atomic force and piezoforce imaging reveal the different orientations of directional order parameters and domain wall character, providing a physical playground for graph theory. Like other counterpart materials ${ }^{16,17}$, ferroelectric domain walls in $\mathrm{Ca}_{3} \mathrm{Ti}_{2} \mathrm{O}_{7}$ are anisotropic and more conducting than their surroundings-although the domains themselves are insulating ${ }^{19,20,24}$. Theoretical modeling and electron diffraction experiments recently revealed that polarization rotates across the ferroelectric walls in a manner that is more Néel than Ising-like ${ }^{25}$. Ferroelastic walls in $\mathrm{Ca}_{3} \mathrm{Ti}_{2} \mathrm{O}_{7}$ are linear and separate different structural twin domains ${ }^{2 \sigma}$. Because the boundaries connect domains with different spontaneous strain states, mechanical compatibility conditions determine their orientation ${ }^{24,26,27}$. These ferroelastic walls are also $90^{\circ}$ ferroelectric walls. Within each twin, there also are meandering $180^{\circ}$ ferroelectric walls ${ }^{20}$.

There is considerable interest in the dynamics of domain walls. Broadband scanning impedance microscopy of hexagonal $\mathrm{ErMnO}_{3}$ reveals a domain wall response dominated by bound charge oscillations rather than free carrier conduction ${ }^{28}$. Secondharmonic generation spectroscopies also provide insight into local symmetries and chemical structure and, at the same time, are sensitive imaging tools ${ }^{8}$. X-ray photon correlation spectroscopy has uncovered ferroelectric domain wall dynamics in $\mathrm{PbZr}_{0.55} \mathrm{Ti}_{0.45} \mathrm{O}_{3}$ as well ${ }^{29}$. Despite a number of significant discoveries, almost nothing is known about domain wall phonons. Infrared spectroscopy, a powerful tool for probing phonons and their symmetries, has been unable to address the nano-scale heterogeneities of domain walls due to its poor spatial resolution. The long wavelengths of infrared light necessarily mean that, even for a diffraction-limited beam, traditional infrared techniques average over any micro- and nano-structured character of a target material, thereby reducing the sensitivity to these heterogeneities $^{30}$. The development of near-field spectroscopic techniques has made real space imaging of nano-scale heterogeneity feasible, although most efforts are confined to the middle infrared due to the availability of suitable laser and broadband sour$\operatorname{ces}^{31,32}$. The extension into the far infrared using an acceleratorbased source ${ }^{33}$ enables broadband measurement of the lowfrequency response including phonons. Synchrotron-based nearfield infrared spectroscopy ${ }^{34}$ thus has the potential to unlock the properties of defect states such as domain walls in complex oxides and chalcogenides. Near-field infrared spectroscopy is, of course, quite different than tip-enhanced Raman scattering in that ungerade (odd symmetry) phonon modes, which are crucial to the development of ferroelectricity and other functionalities, can be probed and analyzed.

In order to uncover the behavior of fundamental excitations like phonons at domain walls and to explore the structural distortions that they represent, we performed far infrared synchrotron-based near-field nano-spectroscopy of the local phonon response at domain boundaries in $\mathrm{Ca}_{3} \mathrm{Ti}_{2} \mathrm{O}_{7}$ and compared our findings with theoretical models of how the order parameter evolves across the wall. Analysis of the Ti-O stretching and bending modes across the ferroelastic wall reveals subtle changes in the frequency and matrix element that we relate to the underlying modulation of the crystal structure and to the rotation of the structural order parameters. There is significant width to the residual structural distortion across the walls $(60-100 \mathrm{~nm})$ and semiconducting character. This research opens the door to broadband imaging of heterogeneity in ferroics and represents a first step to revealing the rich dynamics of domain walls in these systems. At the same time, it provides crucial information for the development of ultra-low-power devices, switches, polarizers, and computing architectures based upon domain walls ${ }^{35,36}$. Loss mechanisms involving phonons are also key to controlling decoherence in domain wall-based computing architectures ${ }^{28,37}$.

\section{Results}

Structure and order parameters across the domain boundary. The structure of $\mathrm{Ca}_{3} \mathrm{Ti}_{2} \mathrm{O}_{7}$ consists of slabs that contain two layers of $\mathrm{CaTiO}_{3}$ perovskite separated by $\mathrm{CaO}$ rocksalt layers. At room temperature, $\mathrm{Ca}_{3} \mathrm{Ti}_{2} \mathrm{O}_{7}$ crystallizes in the orthorhombic polar space group $\mathrm{A2}_{1} \mathrm{am}^{38,39}$. This structure (Fig. 1a) decomposes into three distinct distortions: an out-of-phase octahedral tilt $\left(a^{-} a^{-} c^{0}\right.$ in Glazer notation $\left.{ }^{40}\right)$ that transforms like the $X_{3}^{-}$ irreducible representation of the high-symmetry prototype structure $I 4 / \mathrm{mmm}$, an in-phase octahedral rotation $\left(a^{0} a^{0} c^{+}\right.$in Glazer notation) that transforms as $X_{2}^{+}$, and a polar distortion that transforms like $\Gamma_{5}^{-}$. Each distortion is represented by a structural order parameter with amplitude $Q$ and phase $\phi^{24}$. For the chosen setting of the orthorhombic axes relative to the tetragonal axes, the twin domains are labeled by different settings of the space group symbol: $A 2_{1} a m$ (twin A) and $B b 2_{1} m$ (twin B). Figure $1 \mathrm{~b}$ shows their structure. In twin $\mathrm{A}$, the $X_{3}^{-}$tilt axis (and polarization direction) lie along [-110], whereas they lie along [110] in twin B. The $X_{2}^{+}$rotation axis lies along [001] in both twins, but the adjacent perovskite slabs have different rotation senses: in twin A, the rotations in the two slabs are in-phase (red arrows), whereas in twin B, they are out of phase. The boundaries connect domains with different spontaneous strain states, so mechanical compatibility conditions determine their orientation $^{27}$. The wall symmetry is obtained by combining the symmetry elements of the two domains and those transforming one domain into the other. To go from twin A to twin B, all three structural order parameters rotate (change phase $\phi$ ) by $90^{\circ}$ (Fig. 1b, c). Thus the $X_{3}^{-}$tilt axis rotates by $90^{\circ}$, whereas the sense of the $X_{2}^{+}$rotation reverses in every other perovskite slab. At the center of the domain wall, the local structure is $C 2 \mathrm{~mm}$ (Fig. 1b), where the $X_{3}^{-}$tilt axes in slabs 1 and 2 are perpendicular to each other (tilts about [100] and [010] axes in slabs 1 and 2, respectively). The $X_{2}^{+}$rotation is unchanged in slab 1 and zero in slab 2. Thus the amplitude of the $X_{3}^{-}$order parameter remains relatively constant across the domain wall, whereas the $X_{2}^{+}$order parameter amplitude is suppressed in the middle of the path (Fig. 1d, e). Whether local $\mathrm{C} 2 \mathrm{~mm}$ structure is realized at the center depends on wall width. If it is atomically thin, the structural change from one domain to the other will be abrupt, but if (as observed here) the walls are wide, a macroscopic region with $C 2 \mathrm{~mm}$ symmetry may be realized.

Locating domain walls in different fields of view. Figure 2 summarizes the character of the different ferroelastic and ferroelectric domain walls in $\mathrm{Ca}_{3} \mathrm{Ti}_{2} \mathrm{O}_{7}$ at room temperature. The ferroelastic $\left(90^{\circ}\right.$ ferroelectric) walls are apparent under cross-polarized light and readily identified by a color change as linear twin boundaries in an optical microscope as illustrated in Supplementary Fig. 1. They appear as faint parallel lines in higher-magnification images. These features are present in a field of view where we have both atomic force microscopic (AFM) topography and near-field infrared spectroscopy. Nano-spectroscopic line scans (where a spectrum is acquired at each pixel) are set up accordingly to cross ferroelastic walls of interest. The line scans discussed here are 
a

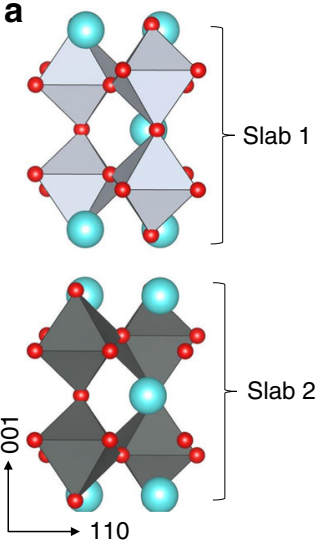

d

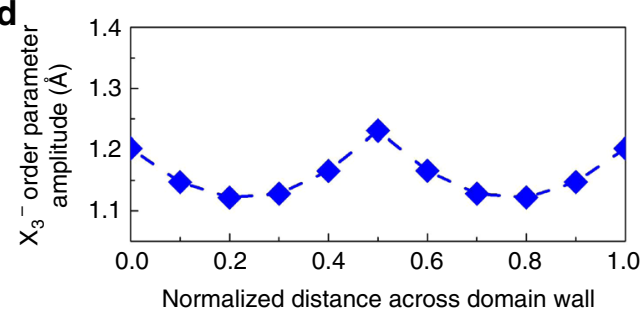

b

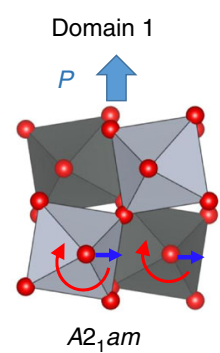

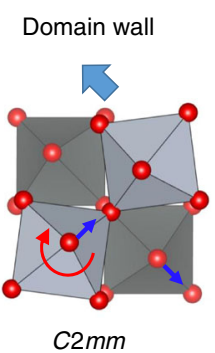

$\mathrm{C} 2 \mathrm{~mm}$
Domain 2

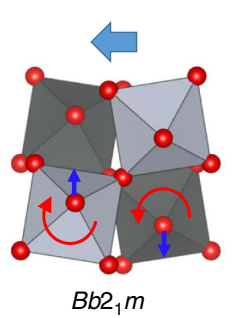

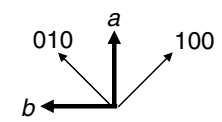

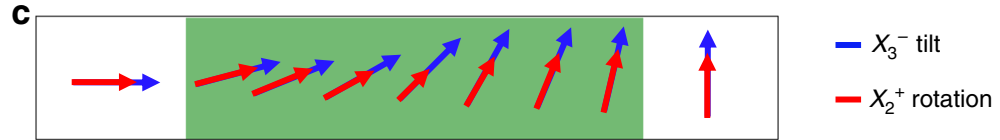

Fig. 1 Bulk crystal and ferroelastic domain wall structure. a Crystal structure of $\mathrm{Ca}_{3} \mathrm{Ti}_{2} \mathrm{O}_{7}$ (space group $A 2_{1}$ am). The two CaTiO 3 perovskite slabs are shown in light and dark gray, with the Ca cations in light blue. The structure of the two orthorhombic twin domains is shown in $\mathbf{b}$ real space and $\mathbf{c}$ order parameter space. The order parameters for the $X_{3}^{-}$octahedral tilt and $X_{2}^{+}$octahedral rotation are shown with blue and red arrows, respectively, in c and the corresponding atomic motions are highlighted using the same colors in $\mathbf{b}$. The bold black arrows show the setting of the orthorhombic relative to the tetragonal axes. The rotation direction of the $\Gamma_{5}^{-}$order parameter is indicated by the arrows with a $P$, indicating polarization direction, above the structures. Upon crossing a ferroelastic domain wall, the octahedral rotation order parameters rotate by $90^{\circ}$; at the midpoint, the local structure is space group $C 2 m m$. d, e The order parameter amplitudes are plotted as a function of the normalized wall width. These amplitudes (reported for a $Z=2$ cell) were obtained using density functional theory calculations of a trajectory through the bulk energy surface. The units of these structural order parameters are that of distance (in $\AA$ ) because they are measuring the amount of distortion-which is calculated by adding up how much all of the atoms in the unit cell are displaced from their high symmetry positions. See Supplementary Information for details

indicated with arrows in Fig. 2a, b. We also identified candidate $180^{\circ}$ ferroelectric walls for analysis and near-field line scans using a combination of AFM, piezoforce microscopy, and a careful examination of the ridges and topography of the crystal surface as illustrated in Fig. 2c, d. These walls are much more challenging to locate because they meander between twin boundaries. A detailed explanation of this process and a summary of our spectroscopic findings are available in Supplementary Information.

Near-field imaging of ferroelastic domain wall phonons. Figure $3 \mathrm{a}, \mathrm{b}$ displays the near-field scattering amplitude $A(\omega)$, which encodes the sample dielectric response across two different ferroelastic domain walls in the hybrid improper ferroelectric $\mathrm{Ca}_{3} \mathrm{Ti}_{2} \mathrm{O}_{7}$. Each contour plot shows the line scan distance (the exact position of which is shown in Fig. $2 \mathrm{a}, \mathrm{b}$ ) vs. frequency, with the color scheme indicative of near-field amplitude. We selected these particular scans to illustrate typical wall variations. The features in $A(\omega)$ are in reasonable agreement with the far-field spectra (Supplementary Information).

A group theoretical analysis shows 72 zone-center phonons that transform as

$$
\Gamma=19 A_{1} \oplus 17 A_{2} \oplus 19 B_{1} \oplus 17 B_{2} .
$$

The $A_{1}, B_{1}$, and $B_{2}$ modes are infrared and Raman active, whereas the $A_{2}$ modes are Raman active only. By considering the atomic displacement patterns arising from the phonon eigenvectors, the broad structures with maxima at 645 and $450 \mathrm{~cm}^{-1}$ are assigned as $\mathrm{Ti}-\mathrm{O}$ stretching and $\mathrm{Ti}-\mathrm{O}-\mathrm{Ti}$ bending modes, respectively. In order to associate these localized vibrations with the rotations and tilts that are crucial for the trilinear coupling and ferroelectric switching, we projected out the character of each calculated phonon. This process is described in detail below. We find that the Ti-O stretching mode has a component that transforms as $X_{2}^{+}$, whereas the Ti-O-Ti bending mode transforms primarily as $X_{3}^{-}$. Thus the symmetry of long-range rotations and tilts are mapped onto the more localized vibrations that are available in our experimental energy window.

Strikingly, the near-field infrared spectrum is sensitive to the ferroelastic $\left(90^{\circ}\right.$ ferroelectric) domain walls. Focusing first on Fig. 3a, we see that this line scan crosses a twin boundary. Both spectral amplitude and line shape are altered across the wall. This is interesting and important because domain walls in perovskites are traditionally considered to be atomically sharp boundaries ${ }^{41}$. Turning to the second set of data in Fig. 3b, a near-field scan over an independent ferroelastic domain wall again reveals a significant decrease in phonon amplitude and line width. There are also very slight frequency shifts (toward the blue) that are at the limit of our resolution. We therefore see that, while the height and width of the wall vary somewhat, the general spectral characteristics are similar.

Point spectra taken from the contour data (Fig. 3c) unveil a more traditional spectral view of the ferroelastic wall, which we can compare to that of the surrounding domain. In order to highlight spectral changes between the wall and the surroundings, we calculated a difference spectrum as $\Delta \bar{A}(\omega)=$ $\bar{A}(\omega)_{\text {DW2 }}-\bar{A}(\omega)_{\text {Domain }}$. This quantity, which reveals average changes (indicated by overbars) in the near-field amplitude, is shown in the upper part of Fig. 3c. We find that the wall phonons 

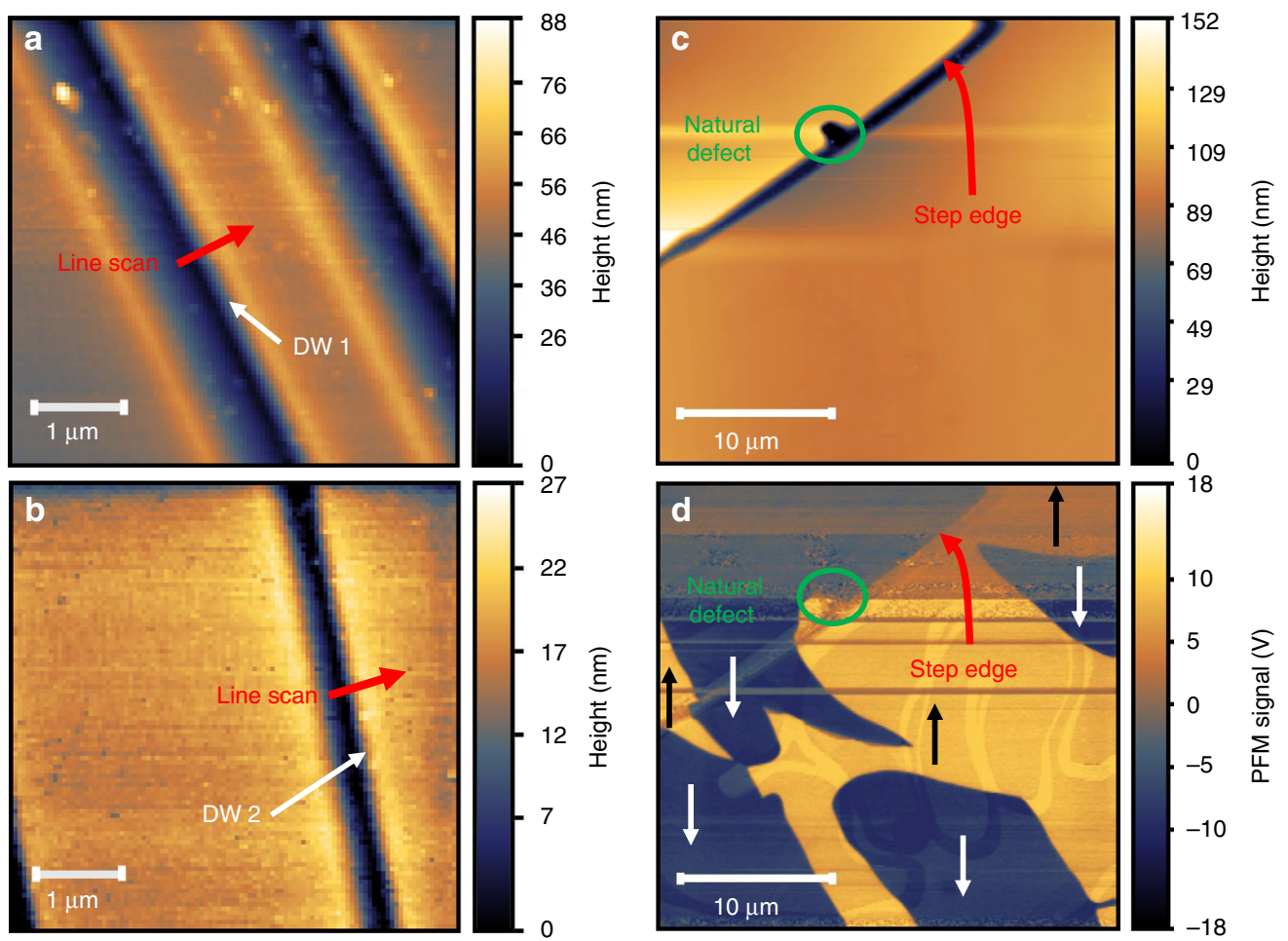

Fig. 2 Combining scanning techniques to locate domain walls. a, b Atomic force microscopic (AFM) images of the crystal surfaces showing the two ferroelastic domain walls of interest (at the edges of the dark blue stripes). These ferroelastic walls separate domains of different spontaneous strain and are also $90^{\circ}$ ferroelectric walls. DW 1 and DW 2 refer to domain walls 1 and 2 . Red arrows indicate direction and path of the line scans. The nanospectroscopic line scans are taken perpendicular to the wall, and the contact angle from one domain to another is $90^{\circ}$. c AFM topography of a smooth area near an identified surface defect (indicated by a green circle) and step edge of approximately $100 \mathrm{~nm}$ height (indicated with a red arrow) compared with d the piezoresponse force microscopic (PFM) image of the same area revealing the placement and orientation of the $180^{\circ}$ ferroelectric domains, indicated by yellow $(+)$ or blue $(-)$ regions with black or white arrows to indicate the polarization direction. All of these structures are present at room temperature

have reduced amplitude and a slight blue shift. The frequency shift is at the limit of our sensitivity, which unfortunately precludes a more detailed analysis. In any case, the blue shift suggests that there is a spontaneous strain across the wall ${ }^{27}$. The strain across the wall occurs because a ferroelastic wall connects domains with different spontaneous strain states; this strain together with the modulation of order parameter amplitudes determines the local wall structure. Another property of interest is conductivity. We immediately notice that the twin boundary is semiconducting rather than metallic because there are strong phonons with no hint of a Drude response. Therefore, the ferroelastic walls are not metallic in the conventional sensealthough they may be slightly more conducting than their surroundings. To verify this observation, we calculated the band gap for the bulk $C 2 \mathrm{~mm}$ structure (the hypothetical structure realized at the midpoint of the domain wall). We find that the computed gap is $0.26 \mathrm{eV}$ less than that of the $A 2{ }_{1}$ am structurenot even close to closing the $3.94 \mathrm{eV}$ gap in this system ${ }^{42}$. This confirms that the ferroelastic walls remain insulating.

Constant frequency cuts in the range of the Ti-O stretching and Ti-O-Ti bending modes uncover another surprising aspect of the walls (Fig. 3d). We take these cuts of the contour data at 460 and $640 \mathrm{~cm}^{-1}$, where the change in near-field amplitude between the wall and the surrounding domain as quantified by $\Delta \bar{A}(\omega)$ in Fig. 3c is strongest. Rather than an atomically sharp boundary, this direct and microscopic probe of the ungerade modes suggests that there is a significant width to the structural distortion. We find widths from 60 to $100 \mathrm{~nm}$ for the range of domain walls investigated. This is akin to the length scale of structural relaxations in strained epitaxial thin films. The four different types of ferroelastic domain walls in
$\mathrm{Ca}_{3} \mathrm{Ti}_{2} \mathrm{O}_{7}$ (head-to-head, head-to-tail, tail-to-head, and tail-to-tail) ${ }^{20}$ along with the prediction from Landau theory that charged walls are thicker than their neutral counterparts ${ }^{43}$ provide a natural explanation for this variation.

Relating near-field amplitude to the order parameters. We now consider how localized phonons map onto the underlying longrange structural order parameters in Fig. 1d, e. We know that the $19 A_{1}$ phonons maintain $A 2_{1}$ am crystal symmetry across the wall, so we can loosely think of the $A_{1}$ phonons as excitations of the structural order parameters. Since distortions that transform like the $X_{3}^{-}, X_{2}^{+}, \Gamma_{5}^{-}$, and $\Gamma_{1}^{+}$irreducible representations of $I 4 / \mathrm{mmm}$ contribute to the $A 2_{1} a m$ structure, each $A_{1}$ phonon can (in principle) excite a mixture of these four structural order parameters. We therefore performed a change of basis and projected the computed $A_{1}$ phonon eigenvectors $\mathbf{e}_{A_{1}}$ onto a basis of symmetry-adapted modes $\mathbf{e}_{i \tau}$ that transform like the irreducible representation $\tau=\left\{\Gamma_{1}^{+}, \Gamma_{5}^{-}, X_{3}^{-}, X_{2}^{+}\right\}$of the high-symmetry prototype structure $I 4 / \mathrm{mmm}$. Figure 4 a displays the results of this projection. Details are in Supplementary Information.

Remarkably, certain phonons almost completely overlap with a single symmetry-adapted mode-exciting only one structural order parameter-whereas, others are a mixture. Focusing first on the low-frequency phonons with calculated frequencies 428 and $466 \mathrm{~cm}^{-1}$, we find that they transform primarily as $\mathbf{e}_{X_{3}^{-}}$. These phonons are $\mathrm{Ti}-\mathrm{O}-\mathrm{Ti}$ bends although they involve different bond angles (Fig. 4b, c). We next consider the phonons calculated to be at 652 and $546 \mathrm{~cm}^{-1}$. They primarily overlap with $\mathbf{e}_{\Gamma_{1}^{+}}$and $\mathbf{e}_{X_{2}^{+}}$. The atomic displacement patterns (Fig. $4 \mathrm{~d}$, e) reveal that the $652 \mathrm{~cm}^{-1}$ 

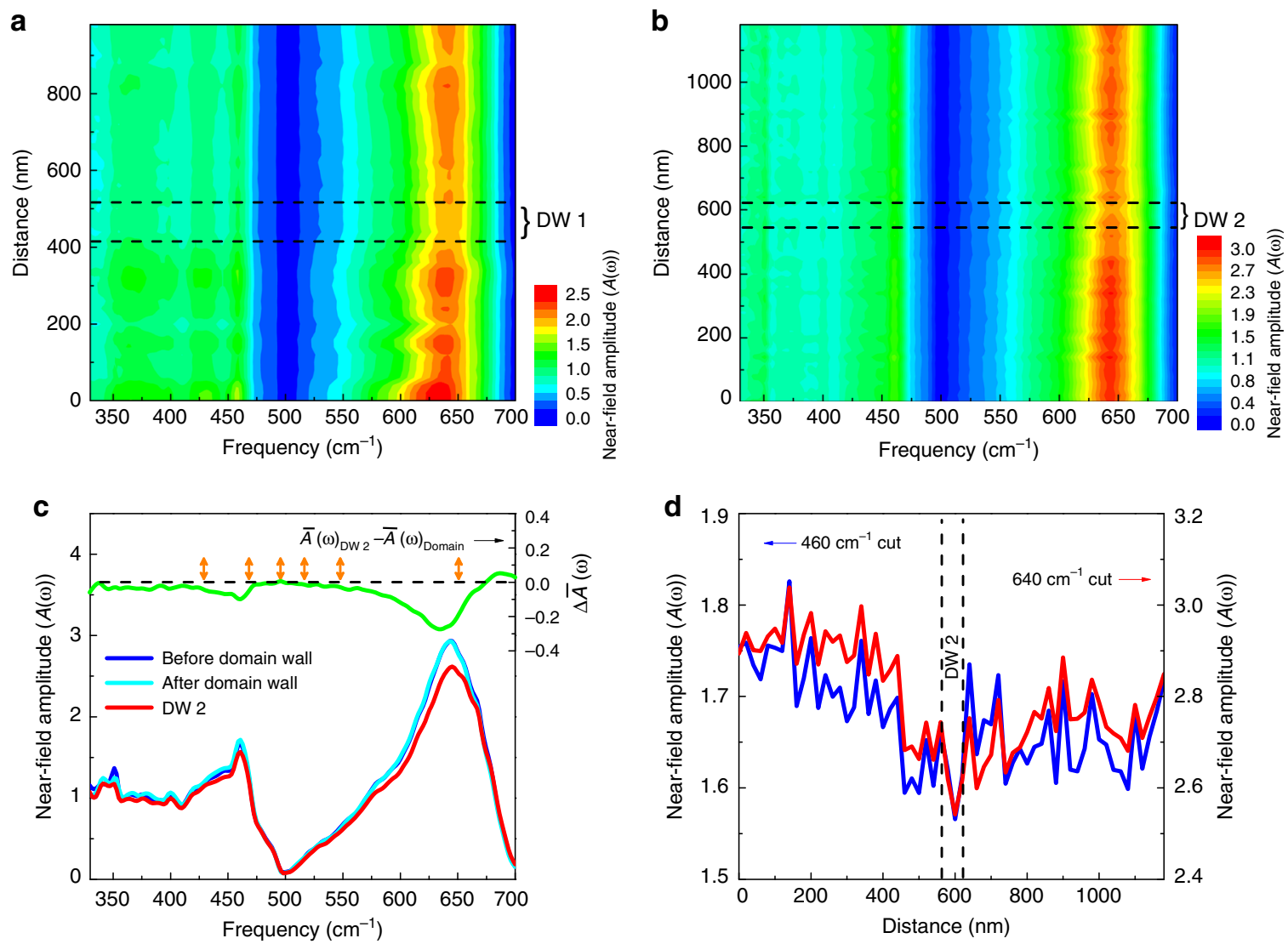

Fig. 3 Near-field infrared spectroscopy of $\mathrm{Ca}_{3} \mathrm{Ti}_{2} \mathrm{O}_{7}$. $\mathbf{a}$, b Contour plots of the near-field amplitude normalized to a gold reference across two different domain walls as indicated in Fig. 2a, b. We label these walls as DW 1 and DW 2. The step size is $20 \mathrm{~nm}$, and the tip resolution is $20 \times 20 \mathrm{~nm}{ }^{2}$. The black dashed lines indicate the domain wall locations, and the brackets denote effective wall widths. $\mathbf{c}$ Fixed distance cuts of the contour spectra in $\mathbf{b}$ show $A(\omega)$ at the ferroelastic domain wall compared with two different point scans away from the wall. Average changes in the near-field amplitude, calculated as $\Delta \bar{A}(\omega)=\bar{A}(\omega)_{\text {DW2 }}-\bar{A}(\omega)_{\text {Domain }^{\prime}}$ reveal the difference and, at the same time, reduce the noise. Here the bars denote an average response. This analysis demonstrates that wall phonons have reduced amplitude and a slight blue shift. The six $A_{1}$ modes within the experimental energy window are indicated by double-sided orange arrows. d Fixed frequency cuts of the contour data in $\mathbf{b}$ showing how intensity at 460 and $640 \mathrm{~cm}^{-1}$ varies across DW 2 . According to the calculation of $\Delta \bar{A}(\omega)$ in $\mathbf{c}$, these frequencies are most sensitive to the presence of the domain wall. They are also very near the calculated $A_{1}$ symmetry vibrational modes (shown as orange arrows in $\mathbf{c}$ )

phonon is a $c$-polarized Ti-O stretch, whereas the $546 \mathrm{~cm}^{-1}$ mode is an $a b$-polarized Ti-O stretch. This motivates our assignment of the broad experimental structure centered at $645 \mathrm{~cm}^{-1}$ as bond stretching. The width of the spectral peak suggests that the $a b$-plane and $c$-directed Ti-O stretching modes overlap.

There are three distinct types of oxygen centers in the layered Ruddlesden-Popper structure. These include the equatorial oxygens $\mathrm{O}_{\text {eq }}$, as well as two different types of apical oxygens: those that border the rocksalt layer $\mathrm{O}_{\mathrm{RS}}$ and those that lie in the middle of the perovskite slab, $\mathrm{O}_{\mathrm{p}}$. We find that the $466 \mathrm{~cm}^{-1}$ phonon is primarily a Ti- $\mathrm{O}_{\mathrm{P}}$-Ti bend, whereas the $428 \mathrm{~cm}^{-1}$ mode involves both $\mathrm{Ti}-\mathrm{O}_{\mathrm{RS}}-\mathrm{Ti}$ and $\mathrm{Ti}-\mathrm{O}_{\text {eq }}$-Ti bending motion. From this analysis, we conclude that the broad experimental peak centered near $645 \mathrm{~cm}^{-1}$ contains excitations that transform like $X_{2}^{+}$and $\Gamma_{1}^{+}$, whereas the wide experimental feature centered near $450 \mathrm{~cm}^{-1}$ in the near-field spectrum transforms primarily as the $X_{3}^{-}$irreducible representation.

Order parameter trends vs. near-field response of ferroelastic domain walls. In order to test the correspondence between these symmetry objects, we plotted the near-field amplitude of the 460 and $640 \mathrm{~cm}^{-1}$ phonons as a function of distance across the ferroelastic domain wall and overlaid predictions for how the order parameters change across the wall. Importantly, these two frequencies are most sensitive to the presence of the domain wall, and they are very near the calculated $A_{1}$ mode positions (Fig. 3c). The agreement, while not perfect, has several striking aspects. Our model predicts that the $X_{3}^{-}$amplitude is relatively constant across the wall, whereas the amplitude of the $X_{2}^{+}$rotation changes significantly and is suppressed in the center. Domain wall 1 exhibits reasonable overall agreement with these predictions (Fig. 5a, b). The $460 \mathrm{~cm}^{-1}$ feature remains relatively constant across the wall, although the anticipated minimum in the fixed frequency scan at $640 \mathrm{~cm}^{-1}$ is not well pronounced. Domain wall 2 is different (Fig. 5c, d), illustrating what we have found to be typical variations. The fixed frequency near-field scan at $460 \mathrm{~cm}^{-1}$ is relatively flat across the wall-in agreement with the predictions of the $X_{3}^{-}$ order parameter. At the same time, the $640 \mathrm{~cm}^{-1}$ feature is suppressed at the center of the wall, as anticipated. Overall, both $X_{3}^{-}$ and $X_{2}^{+}$track the behavior of the walls fairly well albeit with some deviation. Therefore, we can loosely but not completely think of the long-range rotations and tilts as mapping onto the more localized vibrations that are available in our experimental energy window. Mixing effects (Fig. 4a) and signal-to-noise issues are the primary reasons that the agreement is not better.

This order parameter framework provides appealing insight into the spatial extent of the ferroelastic domain walls in 

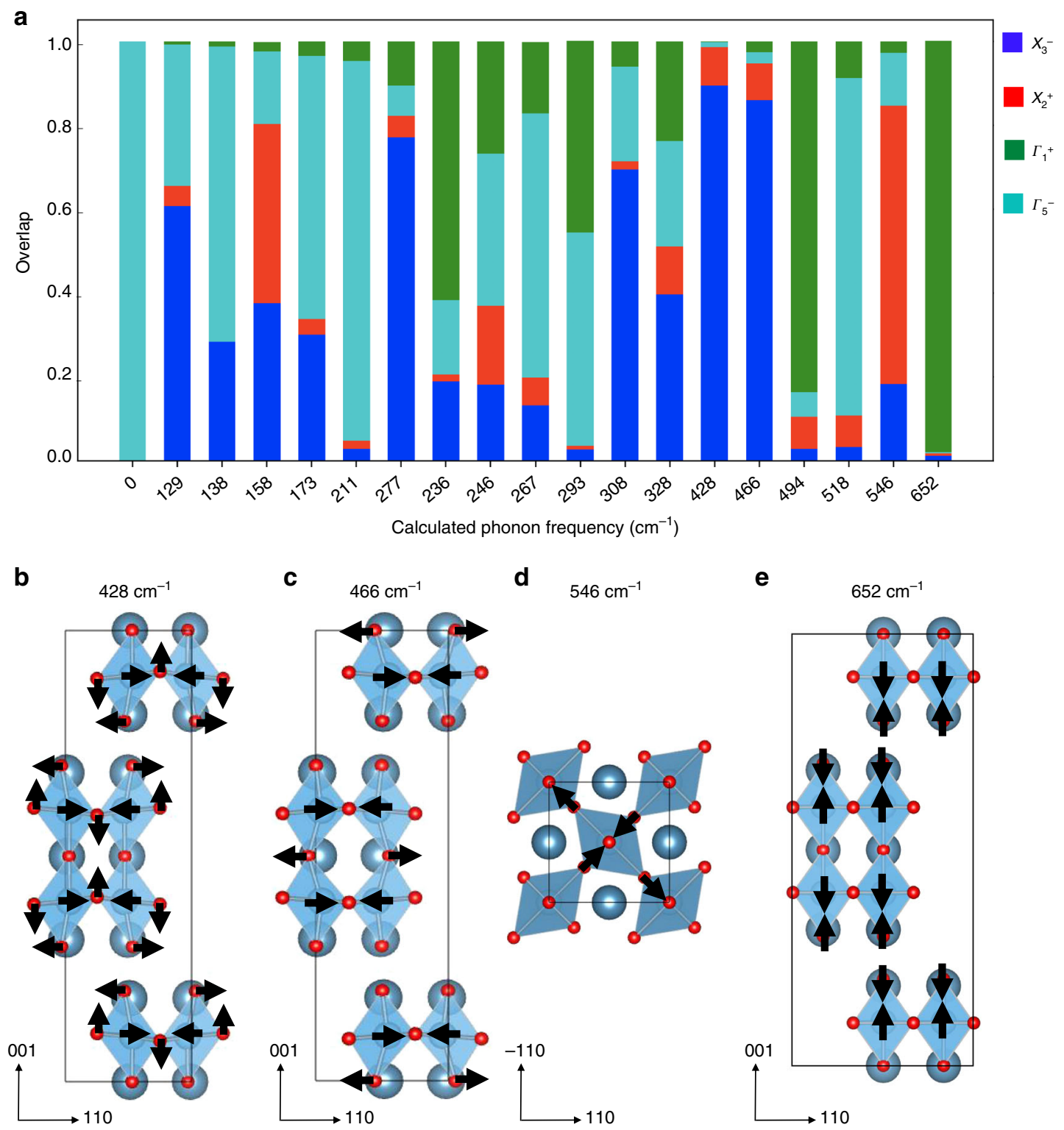

Fig. 4 Phonon overlaps and calculated displacement patterns. a The $19 A_{1}$ symmetry phonon eigenvectors projected onto the $14 / \mathrm{mmm}$ symmetry-adapted modes. The labels are the calculated phonon frequencies. b-e Atomic displacement patterns of four selected phonons from $\mathbf{a}$. The black arrows display the largest atomic motions within each displacement pattern. The 428 and $466 \mathrm{~cm}^{-1}$ phonons largely consist of Ti-O-Ti bond angle bends. The 546 and $652 \mathrm{~cm}^{-1}$ phonons are bond-stretching modes in the $a b$ plane and along the $c$-axis, respectively

$\mathrm{Ca}_{3} \mathrm{Ti}_{2} \mathrm{O}_{7}$. Twin boundaries are traditionally considered to be quite narrow ${ }^{41,44}$, although recent work suggests that they may be wider than previously supposed ${ }^{45}$. In systems with octahedral rotations, the width and energy of a ferroelastic wall depends on the orientation of the octahedral rotation axes with respect to the domain wall plane $e^{41,46}$. In particular, symmetry constraints that require the octahedral rotation amplitude to go to zero at the center of the wall can increase the wall's width. In $\mathrm{Ca}_{3} \mathrm{Ti}_{2} \mathrm{O}_{7}$, as the $X_{2}^{+}$order parameter rotates by $90^{\circ}$ across the ferroelastic wall, symmetry dictates that the $a^{0} a^{0} c^{+}$rotation turns off in every other perovskite slab (Fig. 1c). The spatial extent of the ferroelastic walls-ranging from 60 to $100 \mathrm{~nm}$ in our measurements-may therefore originate from frustration of the $X_{2}^{+}$ rotation. In addition to the octahedral rotation order parameters discussed here, the spontaneous strain also changes across the ferroelastic wall. This means that the elastic properties also play a role in determining the domain wall width and energy ${ }^{27,47}$. Based on experimentally reported room temperature lattice parameters ${ }^{38}, \mathrm{Ca}_{3} \mathrm{Ti}_{2} \mathrm{O}_{7}$ has a small orthorhombic distortion with spontaneous strain $\eta=(b-a) /(b+a)=8 \times 10^{-4}$. The elastic energy per area of a ferroelastic wall can be estimated as $\eta^{2} C d$, where $C$ is the elastic stiffness coefficient and $d$ is the domain wall width. Taking $C$ from density functional theory (DFT) calculations $^{48}$ and estimated wall widths from our experiments, we find the elastic energy per area to be roughly $10 \mathrm{~mJ} / \mathrm{m}^{2}$. This relatively small elastic energy contribution may make wide walls a favorable situation.

\section{Discussion}

In this work, we combine synchrotron-based infrared nanospectroscopy and theoretical modeling to unlock a nano-scale view of ferroelastic domain walls in hybrid improper ferroelectric $\mathrm{Ca}_{3} \mathrm{Ti}_{2} \mathrm{O}_{7}$. While domain walls have long been known to play a 
Domain wall 1
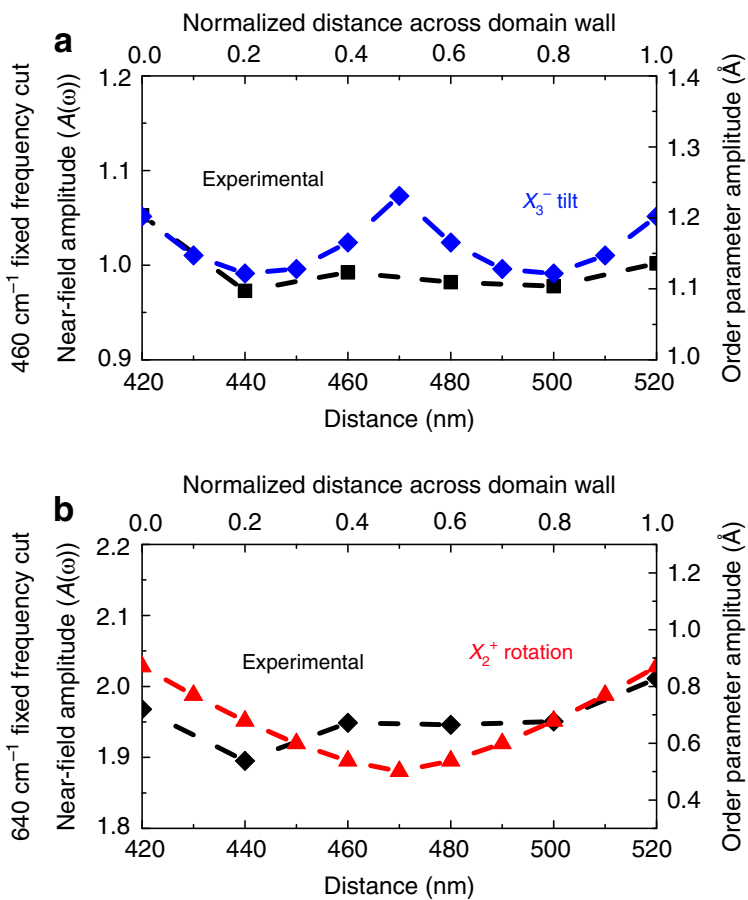

Domain wall 2
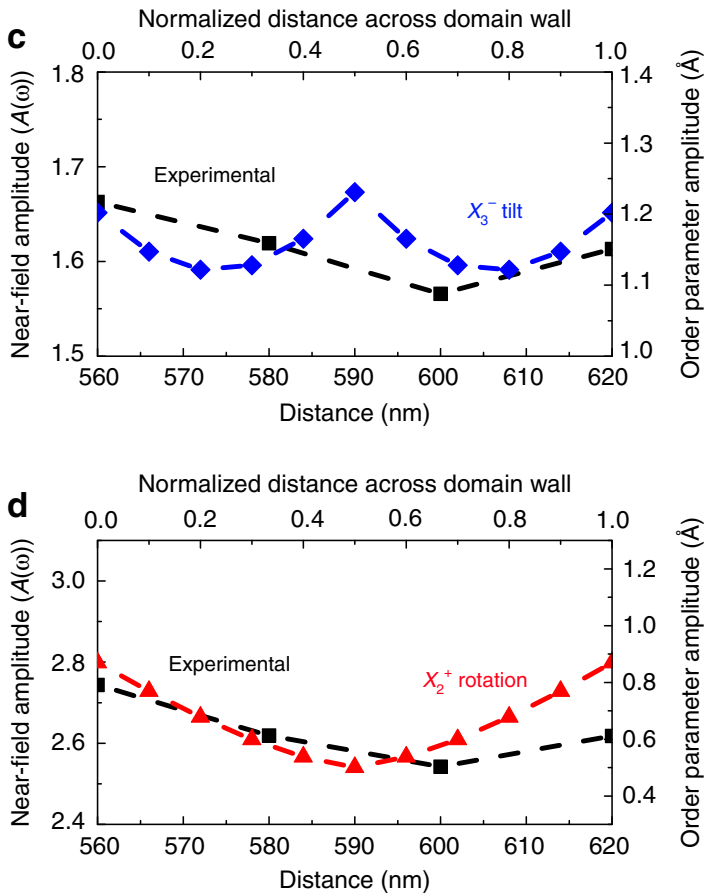

Fig. 5 Order parameter trends across the structural domain walls. a-d Comparison of predicted $X_{3}^{-}$(tilt, blue diamonds) and $X_{2}^{+}$(rotation, red triangles) order parameters with the measured near-field amplitude at 460 and $640 \mathrm{~cm}^{-1}$ across the two walls of interest from Fig. 3a, b. Both the normalized distance across each wall (treated here as a scalable parameter) as well as the actual line scan positions (determined from the near-field response) are shown. The microscopic distortions across the wall relax more slowly than topography would suggest

key role in determining the functionality of ferroic materials, exploration of the atomic- and nano-scale structure and properties of these walls is in its early stages, enabled by new highresolution imaging techniques and complementary theoretical methods. In particular, we elucidate how the phonon response evolves across a $90^{\circ}$ ferroelastic wall and relate it to the underlying structural changes that occur within the wall. We find that these twin boundaries have a surprisingly large spatial extent, suggesting that ferroelastic walls are not always narrow objects.

\section{Methods}

Crystal growth and scanning techniques to locate domain walls. High-quality single crystals of $\mathrm{Ca}_{3} \mathrm{Ti}_{2} \mathrm{O}_{7}$ were synthesized by optical floating zone techniques as described previously ${ }^{20}$. Surface topography was scanned using AFM, and ferroelastic domain walls were identified using a combination of visual inspection of orthorhombic twin boundaries and cross-polarized light. We also carried out piezoforce microscopy to identify ferroelectric domains and walls. Identification of common step edges and defects allow these images to be overlaid-even though they are not in the same field of view. In this manner, we are able to navigate around the surface and examine the lattice dynamics across different ferroelastic and ferroelectric domain walls.

Synchrotron-based near-field infrared spectroscopy. Near-field infrared spectroscopy was performed using the set-ups at beamlines 5.4 and 2.4 at the Advanced Light Source, Lawrence Berkeley National Laboratory ${ }^{34}$. The apparatus is configured as an asymmetric Michelson interferometer, in which one arm consists of an AFM (Bruker Innova or Neaspec neaSNOM) and the other arm is a moving mirror. Synchrotron infrared light is focused and scattered off an AFM tip in close proximity to the sample surface. The scattered light is combined with the reference beam from the moving mirror on a silicon or KRS- 5 beamsplitter and detected by a liquid helium-cooled Ge:Cu detector. Scanning the mirror of the reference arm creates an interferogram, which is Fourier-transformed to obtain both amplitude and phase information, which is related to the real and imaginary parts of the optical dielectric function of the material. The incident light is $p$-polarized with respect to the sample such that the polarization is parallel to the tip axis. This configuration enables the most efficient coupling to the antenna modes of the tip, but there is also a component of polarization in the plane of the sample due to the focusing angle of the off-axis parabolic mirror. However, the strong enhancement of the metal tip localizes and enhances the optical field linearly polarized parallel to the tip axis, such that the technique is most sensitive to phonon and vibrational modes perpendicular to the sample surface ${ }^{49}$. To discriminate the near-field signal from the far-field background signal, the AFM is operated in non-contact (tapping) mode at a typical frequency of $250-300 \mathrm{kHz}$, and the corresponding detector signal is demodulated at twice the tapping frequency. Our measurements were performed with typical free oscillation tip amplitudes in the $80-100-\mathrm{nm}$ range with a setpoint between $70 \%$ and $78 \%$ of the free-tapping amplitude, such that the engaged tapping amplitude values were in the 55-75-nm range. Routine approach curves with free oscillation tip amplitudes in the $80-100-\mathrm{nm}$ range taken on gold indicate a nearfield tip enhancement within $<30 \mathrm{~nm}$ of the surface with little-to-no second-

harmonic signal when the sample is withdrawn further. Different setpoints within the $70-78 \%$ range have minimal-to-no effects on the sample amplitude or shape. The setpoint is typically chosen to have the least damping (i.e., closer to $78 \%$ ) while still maintaining good AFM feedback and reproducibility between the forward and backward traces. This approach minimizes tip wear as well.

Our near-field infrared measurements were carried out at room temperature over a frequency range between 330 and $800 \mathrm{~cm}^{-1}$. The near-field spectra are corrected for the limited transmissivity of the entire set-up by normalizing the power spectrum of the sample to that of a gold reference mirror. Both second- and third-harmonic signals were analyzed. The spatial resolution of this technique is limited by the AFM tip radius, which is typically $<25 \mathrm{~nm}$ for the tips used (Nanosensor PtSi-NCH). Our line scans employed a step size of $20 \mathrm{~nm}$. Multiple line scans across the same domain wall are very similar in character with variances on the order of the noise level. Scans across different domain walls reveal some variations, which is why we show the results for two different walls in this work.

Far-field infrared spectroscopy. We also carried out traditional, far-field spectroscopy using a suite of Fourier transform and grating spectrometers covering the spectral range from 15 to $65,000 \mathrm{~cm}^{-1}$ for comparison with the near-field infrared response. Data were collected in reflectance mode in the $a b$-plane and along the $c$-direction. A summary of mode positions and assignments is available in Supplementary Information.

Symmetry analysis and lattice dynamics calculations. DFT calculations were performed using projector augmented wave pseudopotentials ${ }^{50}$ and the PBEsol functional, as implemented in VASP ${ }^{51}$. We used the (Ca_sv, Ti_sv, O) VASP pseudopotentials with electronic configurations $3 s^{2} 3 p^{6} 4 s^{2}, 3 s^{2} 3 p^{6} 4 s^{2} 3 d^{2}$, and $2 s^{2} 2 p^{4}$, respectively. Calculations were performed in a 48 -atom supercell commensurate with both orthorhombic twins of $\mathrm{Ca}_{3} \mathrm{Ti}_{2} \mathrm{O}_{7}$ with a $6 \times 6 \times 2$

Monkhorst-Pack $k$-point mesh and a $600 \mathrm{eV}$ plane wave cutoff. All structural relaxations were performed with a force convergence tolerance of $2 \mathrm{meV} / \mathrm{A}$. The 
theoretical curves across domain walls were obtained by considering a path through the bulk energy surface connecting the two domains and calculating a sequence of bulk structures along that path. This was done using nudged elastic band calculations, allowing the ions to relax at each image along the path-between the $A 2_{1}$ am domain to the midpoint structure $C 2 \mathrm{~mm}$ and into the $B b 2_{1} \mathrm{~m}$ domain. Note that we are considering a sequence of bulk structures along the path through the bulk energy landscape that connects the two domains; we are not taking into account order parameter gradients and strains, which are known to be important at the domain wall. While this level of theory does not permit quantitative predictions of, e.g., domain wall widths, we find that it places a simple focus on the evolution of the structural order parameters across the domain wall. Phonon frequencies and eigenvectors were calculated using density functional perturbation theory ${ }^{52}$. We performed group theoretic analysis with the aid of ISOTROPY ${ }^{53}$ and the Bilbao Crystallographic Server ${ }^{5-57}$ and visualized crystal structures using VESTA ${ }^{58}$.

\section{Data availability}

Relevant data are available upon request from the corresponding authors, J.L.M. (email: musfeldt@utk.edu) and E.A.N. (email: enowadnick@ucmerced.edu)

Received: 2 February 2019; Accepted: 7 October 2019;

Published online: 20 November 2019

\section{References}

1. Tranquada, J. M., Sternlieb, B. J., Axe, J. D., Nakamura, Y. \& Uchida, S. Evidence for stripe correlations of spins and holes in copper oxide superconductors. Nature 375, 561-563 (1995).

2. Dagotto, E. Complexity in strongly correlated electronic systems. Science 309, 257-262 (2005).

3. Katakura, I. et al. Development of high-speed polarizing imaging system for operation in high pulsed magnetic field. Rev. Sci. Instrum. 81, 043701 (2010).

4. Singh-Bhalla, G., Selcuk, S., Dhakal, T., Biswas, A. \& Hebard, A. F. Intrinsic tunneling in phase separated manganites. Phys. Rev. Lett. 102, 077205 (2009).

5. Liu, M. K. et al. Anisotropic electronic state via spontaneous phase separation in strained vanadium dioxide films. Phys. Rev. Lett. 111, 096602 (2013).

6. Liu, M. et al. Phase transition in bulk single crystals and thin films of $\mathrm{VO}_{2}$ by nanoscale infrared spectroscopy and imaging. Phys. Rev. B 91, 245155 (2015).

7. de Quilettes, D. W. et al. Impact of microsctructure on local carrier lifetime in perovskite solar cells. Science 348, 683-686 (2015).

8. Fiebig, M., Lottermoser, T. \& Meier, D. The evolution of multiferroics. Nat Rev. Mater. 1, 16046 (2016).

9. Scherpereel, D. E., Kazmerski, L. L. \& Allen, C. W. The magnetoelastic interaction of dislocation and ferromagnetic domain walls in iron and nickel. Metall. Mater. Trans. B 1, 517-524 (1970).

10. Seidel, J. et al. Conduction at domain walls in oxide multiferroics. Nat. Mater. 8, 229-234 (2009).

11. Choi, T. et al. Insulating interlocked ferroelectic and structural antiphase domain walls in multerferroic $\mathrm{YMnO}_{3}$. Nat. Mater. 9, 253-258 (2010).

12. Schröder, M. et al. Conducting domain walls in lithium niobate single crystals. Adv. Funct. Mater. 22, 3936-3944 (2012).

13. Yang, J. C., Huang, Y. L., He, Q. \& Chu, Y. H. Multifunctionalities driven by ferroic domains. J. Appl. Phys. 116, 066801 (2014)

14. Ma, E. Y. et al. Charge-order domain walls with enhanced conductivity in a layered manganite. Nat. Commun. 6, 7595 (2015).

15. Stolichnov, I. et al. Bent ferroelectric domain walls as reconfigurable metalliclike channels. Nano Lett. 15, 8049-8055 (2015).

16. Meier, D. et al. Anisotropic conductance at improper ferroelectric domain walls. Nat. Mater. 11, 284 (2012).

17. Mundy, J. A. et al. Functional electronic inversion layers at ferroelectric domain walls. Nat. Mater. 16, 622-627 (2017).

18. Van Aert, S. et al. Direct observation of ferrielectricity at ferroelastic domain boundaries in $\mathrm{CaTiO}_{3}$ by electron microscopy. Adv. Mater. 24, 523-527 (2012).

19. Huang, F.-T. et al. Domain topology and domain switching kinetics in a hybrid improper ferroelectric. Nat. Commun. 7, 11602 (2016).

20. Oh, Y. S., Luo, X., Huang, F.-T., Wang, Y. \& Cheong, S.-W. Experimental demonstration of hybrid improper ferroelectricity and the presence of abundant charged walls in $(\mathrm{Ca}, \mathrm{Sr})_{3} \mathrm{Ti}_{2} \mathrm{O}_{7}$ crystals. Nat. Mater. 14, 407-413 (2015).

21. Benedek, N. A. \& Fennie, C. J. Hybrid improper ferroelectricity: a mechanism for controllable polarization-magnetization coupling. Phys. Rev. Lett. 106, 107204 (2011)

22. Harris, A. B. Symmetry analysis for the Ruddlesden-Popper systems $\mathrm{Ca}_{3} \mathrm{Mn}_{2} \mathrm{O}_{7}$ and $\mathrm{Ca}_{3} \mathrm{Ti}_{2} \mathrm{O}_{7}$. Phys. Rev. B 84, 064116 (2011).
23. Benedek, N. A., Rondinelli, J. M., Djani, H., Ghosez, P. \& Lightfoot, P. Understanding ferroelectricity in layered perovskites: new ideas and insights from theory and experiments. Dalton Trans. 44, 10544-10558 (2015).

24. Nowadnick, E. A. \& Fennie, C. J. Domains and ferroelectric switching pathways in $\mathrm{Ca}_{3} \mathrm{Ti}_{2} \mathrm{O}_{7}$ from first principles. Phys. Rev. B 94, 104105 (2016).

25. Lee, M.-H. et al. Hidden antipolar order parameter and entangled Néel-type charged domain walls in hybrid imprper ferroelectrics. Phys. Rev. Lett. 119, 157601 (2017).

26. Munro, J. M. et al. Discovering minimum energy pathways via distortion symmetry groups. Phys. Rev. B 98, 085107 (2018).

27. Tagantsev, A. K., Cross, L. E. \& Fousek, J. Domains in Ferroic Crystals and Thin Films. Chapter 2 (Springer, New York, 2010).

28. $\mathrm{Wu}, \mathrm{X}$. et al. Low-energy structural dynamics of ferroelectric domain walls in hexagonal rare-earth manganites. Sci. Adv. 3, el602371 (2017).

29. Gorfman, S. et al. Ferroelectric domain wall dynamics characterized with Xray photon correlation spectroscopy. Proc. Natl Acad. Sci.115, E6680-E6689 (2018)

30. Sun, Q.-C. et al. Spectroscopic signatures of domain walls in hexagonal $\mathrm{ErMnO}_{3}$. Phys. Rev. B 90, 121303 (2014).

31. Huth, F. et al. Nano-FTIR absorption spectroscopy of molecular fingerprints at $20 \mathrm{~nm}$ spatial resolution. Nano Lett. 12, 3973-3978 (2012).

32. Muller, E. A., Pollard, B. \& Raschke, M. B. Infrared chemical nanoimaging: accessing structure, coupling, and dynamics on molecular length scales. J. Phys. Chem. Lett. 6, 1275-1284 (2015).

33. Khatib, O. et al. Far infrared synchrotron near-field nanoimaging and nanospectroscopy. ACS Photonics. 57, 2773-2779 (2018).

34. Bechtel, H. A., Muller, E. A., Olmon, R. L., Martin, M. C. \& Raschke, M. B. Ultrabroadband infrared nanospectroscopic imaging. Proc. Natl Acad. Sci. 111, 7191-7196 (2014).

35. Royo, M. et al. Ferroelectric domain wall phonon polarizer. Phys. Rev. Mater. 1, 051402 (2017). (R)

36. Mittal, S. A survey of techniques for approximate computing. ACM Comput. Surv. 48, 62:1-62:33 (2016)

37. Seijas-Bellido, J. A. et al. A phononic switch based on ferroelectric domain walls. Phys. Rev. B 96, 140101 (2017).

38. Senn, M. S. et al. Negative thermal expansion in hybrid improper ferroelectric Ruddlesden-Popper perovskites by symmetry trapping. Phys. Rev. Lett. 114, 034701 (2015)

39. Elcombe, M. M. et al. Structure determinations for $\mathrm{Ca}_{3} \mathrm{Ti}_{2} \mathrm{O}_{7}, \mathrm{Ca}_{4} \mathrm{Ti}_{3} \mathrm{O}_{10}$, $\mathrm{Ca}_{3.6} \mathrm{Sr}_{0.4} \mathrm{Ti}_{3} \mathrm{O}_{10}$ and a refinement of $\mathrm{Sr}_{3} \mathrm{Ti}_{2} \mathrm{O}_{7}$. Acta Cryst. 47, 305-314 (1991).

40. Glazer, A. M. The classification of tilted octahedra in perovskites. Acta Cryst. 28, 3384-3392 (1972).

41. Cao, W. \& Barsch, G. R. Landau-Ginzburg model of interphase boundaries in improper ferroelastic perovskites of $D_{4 h}^{18}$ symmetry. Phys. Rev. B 41, 4334 (1990).

42. Cherian, J. G. et al. Optical spectroscopy and band gap analysis of hybrid improper ferroelectric $\mathrm{Ca}_{3} \mathrm{Ti}_{2} \mathrm{O}_{7}$. Appl. Phys. Lett. 108, 262901 (2016).

43. Yudin, P. V., Gureev, M. Y., Sluka, T., Taganstsev, A. K. \& Setter, N. Anomalously thick domain walls in ferroelectrics. Phys. Rev. B 91, 060102 (2015). (R).

44. Salje, E. K. H. Ferroelastic materials. Annu. Rev. Mater. Res. 42, 265-283 (2012).

45. Schiaffino, A. \& Stengel, M. Macroscopic polarization from antiferrodistortive cycloids in ferroelastic $\mathrm{SrTiO}_{3}$. Phys. Rev. Lett. 119, 137601 (2017).

46. Xue, F. et al. Orientations of low-energy domain walls in perovskites with oxygen octahedral tilts. Phys. Rev. B 90, 220101 (2014).

47. Cao, W., Barsch, G. \& Krumhansl, J. A. Quasi-one-dimensional solutions for domain walls and their constraints in improper ferroelastics. Phys. Rev. B 42, 6396-6401 (1990).

48. Lu, X.-Z. \& Rondinelli, J. M. Epitaxial-strain-induced polar-to-nonpolar transitions in layered oxides. Nat. Mater. 15, 951 (2016).

49. Muller, E. A. et al. Infrared vibrational nanocrystallography and nanoimaging. Sci. Adv. 2, el601006 (2016).

50. Kresse, G. \& Joubert, D. From ultrasoft pseudopotentials to the projector augmented-wave method. Phys. Rev. B 59, 1758-1775 (1999).

51. Kresse, G. \& Hafner, J. Ab initio molecular dynamics for liquid metals. Phys. Rev. B 47, 558-561 (1993).

52. Baroni, $\mathrm{S}$. et al. Phonons and related crystal properties from density-functional perturbation theory. Rev. Mod. Phys. 73, 515-562 (2001).

53. Stokes, H., Hatch, D. \& Campbell, B. ISOTROPY software suite http://stokes. byu.edu/isotropy.html.

54. Aroyo, M. I. et al. Crystallography online: Bilbao Crystallographic Server. Bulg. Chem. Commun. 43, 183-197 (2011).

55. Aroyo, M. I. et al. Bilbao Crystallographic Server I: databases and crystallographic computing programs. Z. Krist. 221, 15-27 (2006).

56. Aroyo, M. I., A., Kirkov, J., Capillas, J.M., Perez-Mato \& H., Wondratschek Bilbao Crystallographic Server II: representations of crystallographic point groups and space groups. Acta Cryst. A62, 115-128 (2006). 
57. Kroumova, E. et al. Bilbao Crystallographic Server: useful databases and tools for phase transitions studies. Phase Transit. 76, 155-170 (2003).

58. Momma, K. \& Izumi, F. VESTA: a three-dimensional visualization system for electronic and structural analysis. J. Appl. Crystallogr. 41, 653 (2008).

\section{Acknowledgements}

Research at the University of Tennessee is supported by the U.S. Department of Energy, Office of Basic Energy Sciences, Materials Science Division under award DE-FG0201ER45885. Portions of the measurements utilized beamlines 5.4 and 2.4 at the Advanced Light Source, which is a DOE Office of Science User Facility operated under contract no. DE-AC02-05CH11231, including the remote user program from NSLS-II under contract DE-SC0012704. Work at Cornell is supported by the Army Research Office under grant W911NF-10-1-0345. Work at Rutgers is funded by the Gordon and Betty Moore Foundation's EPiQS Initiative through Grant GBMF4413 to the Rutgers Center for Emergent Materials. E.A.N. acknowledges additional support from the New Jersey Institute of Technology. M.B.R. and O.K. acknowledge support from the U.S. Department of Energy, Office of Basic Sciences, Division of Material Sciences and Engineering, under award no. DE-SC0008807. O.K. acknowledges support from the ALS Postdoctoral Fellowship program. Work at Postech is supported by the National Research Foundation of Korea (NRF) funded by the Ministry of Science and ICT (No. 2016K1A4A4A01922028).

\section{Author contributions}

This project was conceived by J.L.M. and K.A.S. Crystals were grown by B.G., C.J.W. and S.-W.C. and piezoforce microscopy was carried out by S.J.L. and S.-W.C. Spectroscopic measurements were performed by K.A.S., S.F., S.N.N., O.K., G.L.C., M.C.M., H.A.B. and J.L.M. Data manipulation and visualization assistance was provided by J.K.K. Firstprinciples calculations and predictions based on a symmetry analysis of the order parameters were carried out by E.A.N. and C.J.F. All authors discussed the data. The manuscript was written by K.A.S., E.A.N., H.A.B. and J.L.M., and all authors commented on the document.

\section{Competing interests}

The authors declare no competing interests.

\section{Additional information}

Supplementary information is available for this paper at https://doi.org/10.1038/s41467 019-13066-9.

Correspondence and requests for materials should be addressed to E.A.N. or J.L.M.

Reprints and permission information is available at http://www.nature.com/reprints

Peer Review Information Nature Communications thanks Petr Yudin and the other anonymous reviewers for their contribution to the peer review of this work.

Publisher's note Springer Nature remains neutral with regard to jurisdictional claims in published maps and institutional affiliations.

(c) (i) Open Access This article is licensed under a Creative Commons Attribution 4.0 International License, which permits use, sharing, adaptation, distribution and reproduction in any medium or format, as long as you give appropriate credit to the original author(s) and the source, provide a link to the Creative Commons license, and indicate if changes were made. The images or other third party material in this article are included in the article's Creative Commons license, unless indicated otherwise in a credit line to the material. If material is not included in the article's Creative Commons license and your intended use is not permitted by statutory regulation or exceeds the permitted use, you will need to obtain permission directly from the copyright holder. To view a copy of this license, visit http://creativecommons.org/ licenses/by/4.0/.

(C) The Author(s) 2019 